\title{
Origin of Diffuse Scattering in Relaxor Ferroelectrics
}

\author{
P. Ganesh ${ }^{1}$, E. Cockayne ${ }^{2}$, M. Ahart ${ }^{1}$, R. E. Cohen ${ }^{1}$, B. \\ Burton $^{2}$, R. J. Hemley ${ }^{1}$, Yang Ren ${ }^{3}$, Wenge Yang ${ }^{3}$, Z-G Ye ${ }^{4}$ \\ 1 Geophysical Laboratory, Carnegie Institution of Washington, \\ 5251 Broad Branch Road, Washington, DC 20015, USA \\ 2 Ceramics Division, Materials Science and Engineering Laboratory, \\ National Institute of Standards and Technology, Gaithersburg, MD 20899 USA \\ 3 Advanced Photon Source, Argonne National Laboratory, Argonne, Illinois 60439, USA \\ 4 Department of Chemistry, Simon Fraser University, Burnaby, BC, V5A 1S6, Canada
}

(Dated: September 1, 2018)

\begin{abstract}
High-pressure and variable temperature single crystal synchrotron X-ray measurements combined with first-principles based molecular dynamics simulations study diffuse scattering in the relaxor ferroelectric system PSN $\left(\mathrm{PbSc}_{1 / 2} \mathrm{Nb}_{1 / 2} \mathrm{O}_{3}\right)$. Constant temperature experiments show pressure induced transition to the relaxor phase at different temperatures characterized by butterfly and rod shaped diffuse scattering around the $\{\mathrm{h} 00\}$ and $\{\mathrm{hh} 0\}$ Bragg spots, respectively. The simulations reproduce the observed diffuse scattering features as well as their pressure-temperature behavior, and show that they arise from polarization correlations between chemically-ordered regions, which in previous simulations were shown to behave as polar nanoregions. Simulations also exhibit radial diffuse scattering (elongated towards and away from $\mathbf{Q}=(000)$ ), that persists even in the paraelectric phase, consistent with previous neutron experiments on $\left(\mathrm{PbMg}_{1 / 3} \mathrm{Nb}_{2 / 3} \mathrm{O}_{3}\right)(\mathrm{PMN})$.
\end{abstract}

Single crystal relaxors have huge electromechanical coupling, and show much promise for ultrasonic transducer applications [1]. They have broad frequency and temperature dependent dielectric maxima, which in the special case of relaxor-ferroelectrics [2, 3] drops to a much lower value below the ferroelectric transition temperature $\left(T_{F E}\right)$. The origin of the relaxor phase has been a topic of intense research for over a decade. From refractive index measurements, Burns et al. [4] suggested that formation of polar clusters below a characteristic temperature, now called the Burns temperature $\left(T_{d}\right)$ [4], gives rise to dielectric dispersion, which was confirmed experimentally [5]. These clusters thought to be a few nanometers in size are called polar nanoregions (PNRs).

Recent X-ray and neutron experiments show characteristic shapes of diffuse scattering in the relaxor phase of several lead based relaxors [6, 7, 8, 9] absent in their paraelectric or ferroelectric phases. The main observed feature is anisotropic diffuse scattering around the Bragg peaks along $<110>$ directions. The ferroelectric phase shows weak streaks similar to $\mathrm{BaTiO}_{3}$ and $\mathrm{KNbO}_{3}$, but rotated by 45 -degrees. The paraelectric phase only shows radial diffuse scattering.

Several hypotheses previously advanced to explain the characteristic shapes [6, 10, 11, 12], invoke some type of artificial anisotropic features such as anisotropic strain, correlated atomic shifts, or domain boundaries to generate the experimentally observed anisotropic diffuse scattering features. Fitting the shapes of diffuse scattering features is not sufficient to uniquely determine the nature of the microstructural feature that give rise to them. Incorporating realistic polarization fluctuations via first-principles derived models provide a basis for clarifying the nature of the PNR in relaxors and experimental diffuse scattering observations provide a critical test for any theoretical model. In this Letter, we clarify the microstructural origin of diffuse scattering features in the relaxor- and the paraelectric- phases of PSN, and related materials, by combining single crystal X-ray diffraction experiments and first-principles-based molecular-dynamics simulations [13]. Our result suggests new pathways to engineer novel materials with superior electro-mechanical properties.

X-ray diffraction was measured on single crystal PSN at beamlines 11-ID-C (0.1077 $\AA)$ and 16-BMD $(0.436926 \AA)$ of the Advanced Photon Sources (Argonne National Laboratory). Single crystals of disordered PSN were grown from high-temperature solutions using a mixture of $\mathrm{PbO}$ and $\mathrm{B}_{2} \mathrm{O}_{3}$ as solvent [14]. A crystal with dimension of $70 \times 70 \times 20$ $\mu \mathrm{m}^{3}$ and oriented along (001) was loaded into a diamond anvil cell (DAC) with Ne as the pressure medium. A ruby chip and a small grain of Au were also loaded for pressure determination (accuracy of $0.2 \mathrm{GPa}$ ). A MAR3450 image plate was used to record oscillation photographs. For low temperature measurements, the DAC was loaded in a He flown cryostat, in which temperature was measured with a thermocouple with an accuracy of $\pm 2 \mathrm{~K}$. Additional experimental details about beamline 11-ID-C and 16-BMD can be found in Refs. [15, 16]. To facilitate measurement of the diffuse scattering signal, the sample was rocked $\pm 6^{\circ}$ in omega. Because of its (001) orientation in a cryostat, only the (hk0) indexed Bragg peaks could be observed.

At ambient pressure, PSN enters the relaxor phase below the Burns temperature $\left(\mathrm{T}_{d} \sim 650 \mathrm{~K}\right)$ and undergoes a ferroelectric transition at $\mathrm{T}_{F E} \sim 365 \mathrm{~K}$. In our experiments at $400 \mathrm{~K}$ (Fig. 1a), we observe the characteristic butterfly and rod shaped diffuse scattering. At $250 \mathrm{~K}$ (Fig. 1b), below $\mathrm{T}_{F E}$, the characteristic diffuse scattering diminishes 


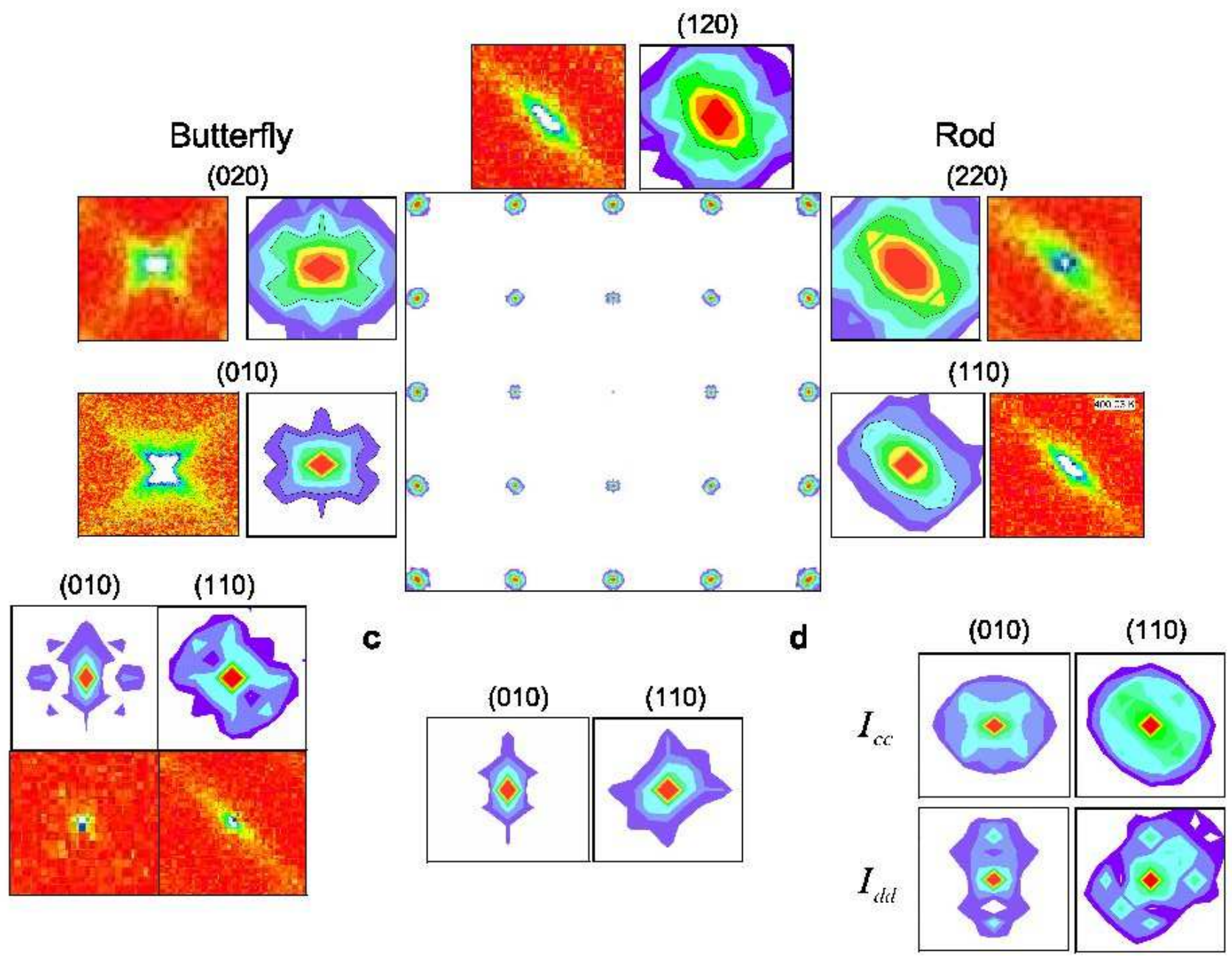

FIG. 1: Temperature dependence of diffuse scattering in (hk0) plane from X-ray diffraction at ambient pressure (red background) and simulations at $\mathrm{P}=18 \mathrm{GPa}$ (white background) (a) Characteristic butterfly and rod shaped diffuse scattering (enhanced intensity along [110] and [110] directions avoiding $\mathbf{Q}=(000))$ is observed in the relaxor phase around $\{\mathrm{h} 00\}$ and $\{\mathrm{hh} 0\}$ Bragg spots, respectively, in experiments $(400 \mathrm{~K})$ and simulations $(200 \mathrm{~K})$ (b) In the ferroelectric phase, the characteristic shapes diminish in experiments $(250 \mathrm{~K})$, similar to simulations $(10 \mathrm{~K})$ (c) In the paraelectric phase, simulations (320 K) only show radial diffuse scattering (d) $I_{c c}$ only shows the butterfly and rod shapes, while $I_{d d}$ shows only the radial patterns in the simulated relaxor phase.

considerably. Diffuse streaks connecting the Bragg peaks grow weaker, yet persist in the ferroelectric phase, similar to those observed in $\mathrm{BaTiO}_{3}$ and $\mathrm{KNbO}_{3}$ (Ref. [17] and references therein).

We observe pressure induced phase transitions at $300 \mathrm{~K}$, where around $1.4 \mathrm{GPa}$ a reappearance of the relaxor phase characterized by diffuse scattering is seen; persisting up to 1.5GPa, but disappearing at 1.8GPa (Fig. 2b). Disappearance of diffuse scattering at higher-pressures indicate a ferroelectric to paraelectric phase transition consistent with dielectric-measurements [2].

At $50 \mathrm{~K}$ (Fig. 2b) we also observe superlattice peaks at low pressures of $\mathrm{P}=0.6 \mathrm{GPa}$ at $(\mathrm{h}+1 / 2 \mathrm{k}+1 / 20)$ suggesting a lowering of symmetry, which reduce in intensity as pressure drives the system to the relaxor phase. Our experiments are on disordered PSN, hence the peaks are not due to ordering [18] but due to possible octahedral rotations. In the relaxor phase, at $2.7 \mathrm{GPa}$, additional satellite peaks with diffuse wings emerge, which persist up to $10 \mathrm{GPa}$. Ordering PNRs with a range of closely spaced incommensurate modulation vectors will give similar satellite spots with diffuse wings.

We performed molecular-dynamics simulations using a model Hamiltonian obtained by expanding the potential energy of PSN about a high symmetry perovskite reference structure and projecting onto the subspace of soft normal 
modes, dominated by $\mathrm{Pb}$ displacements, including the ferroelectric instabilities [19, 20]. Model parameters are fitted to first-principles density functional calculations [21, 22].

The model has chemically ordered regions (COR) with rock-salt ordering of " $\mathrm{Sc}^{+3}$ " and " $\mathrm{Nb}$ " embedded in a chemically disordered region (CDR) that has a random 'B' site configuration. This gives rise to a quenched "random" component to the local electric fields at the Pb-sites. Relative to equal volumes of CDR, polarization is enhanced in CORs, thereby acting as PNRs. The CORs are spatially fixed, because chemical order (disorder) is quenched, but their average polar orientations vary dynamically [21]. The model allows homogeneous strain to fluctuate; inhomogeneous strain, higher-frequency contributions to lattice polarization, and oxygen octahedral tilting are ignored.

In X-ray and neutron scattering experiments, the measured intensity is a Fourier transform of the ensemble averaged two point density-density correlation. Assuming point particles, the intensity is: $I(\mathbf{Q})=<\sum_{\mathbf{i}, \mathbf{j}} e^{\mathbf{i Q} \cdot\left(\mathbf{r}_{i}-\mathbf{r}_{j}\right)}>=<$ $\left|\sum_{i} e^{\mathbf{i} \mathbf{Q} \cdot \mathbf{r}_{i}}\right|^{2}>$ where $i$ runs over the total number of $\mathrm{Pb}$-atoms in the supercell ( $\mathrm{Pb}$ scattering is expected to dominate our experiments as it has a disproportionately large X-ray scattering factor) and $\mathbf{Q}$ values commensurate with the supercell were chosen. Because our distribution of COR in our supercell is not entirely isotropic, we apply full cubic symmetry to the scattering intensity before comparing with experiments.

Fig. 1 shows the temperature dependence of our computed diffuse pattern in the (hk0) plane for the ferroelectric, relaxor and the paraelectric phases at $\mathrm{P}=18 \mathrm{GPa}$. In the relaxor phase (Fig. 1a) we observe the characteristic butterfly and rod shaped diffuse scattering around (h00) and (hh0) Bragg spots, which diminish in their intensity in the ferroelectric phase (Fig. 1b) in excellent comparison to experiments.

Pressure dependent changes in diffuse scattering (Fig. 2a) are similar to those induced by varying temperature. Again, our model captures the experimental pressure induced phase-transition at 300K (Fig. 2b). Experimentally observed streaks, like in $\mathrm{KNbO}_{3}$ have been shown to be due to hopping between equivalent sites [23], and superstructure peaks at $50 \mathrm{~K}$ are possibly due to octahedral rotations, both of which are excluded from our model. But these atomic features do not cause the relaxor phase, which is characterized by the butterfly and rod shaped scattering, which our simple model is able to capture.

As the system is driven towards the paraelectric phase, by increasing temperature or pressure, the characteristic butterfly and rod shapes vanish; however, a weak diffuse pattern that extend radially towards and away from the origin $(\mathbf{Q}=(000))$ persists around all the Bragg peaks. Its intensity is larger in the direction away from the center than towards it, consistent with recent neutron experiments [9] but absent in X-ray due to their low intensity.

We find no significant evolution of the diffraction patterns during our simulations, which access frequencies greater than 10GHz. This suggests only static correlations cause diffuse scattering, consistent with recent experiments on PMN [9].

We find the contributions to the scattering intensity from correlations due to atoms in the different regions (COR and CDR). These are shown in Fig $1 \mathbf{d}$, where $I_{c c}$ and $I_{d d}$ are the intensities obtained by setting CDR and COR Pb displacements to zero respectively. While $I_{c c}$ shows characteristic rod and butterfly features, the radial diffuse pattern is absent. $I_{d d}$ only shows weak radial diffuse pattern. Thus, it is the correlation between COR atoms that give rise to the characteristic diffuse pattern. The $\mathrm{CDR} \mathrm{Pb}$-displacements are dominated by strong electric fields fixed in a "quenched" distribution of "Sc" and "Nb" atoms in the chemically disordered matrix, and therefore radial scattering persists even in the paraelectric phase. This is consistent with experimental observations on PMN [9]. Note that our interpretation that radial diffuse scattering is associated with chemical disorder differs from the interpretation of Gehring et al. that it indicates chemical short range order [9].

To understand the origin of the shapes of the diffuse scattering we write the total intensity as a sum of Bragg and diffuse scattering, and expand the latter in powers of $\xi_{i}=\mathbf{r}_{i}-\mathbf{R}_{0 i}$, which being proportional to the local polarization is a small quantity. The Bragg term is $\sum_{i, j} e^{\mathbf{i} \mathbf{Q} \cdot\left(\mathbf{R}_{0 i}-\mathbf{R}_{0 j}\right)}$, and the lowest order diffuse scattering term is:

$$
I_{d i f f}(\mathbf{G}, \mathbf{q})=<\sum_{i, j}|\mathbf{G}+\mathbf{q}|^{2}\left(\xi_{i} \cdot \hat{\alpha}\right)\left(\xi_{j} \cdot \hat{\alpha}\right) \cos \left(\mathbf{Q} \cdot\left(\mathbf{R}_{\mathbf{0} i}-\mathbf{R}_{\mathbf{0} j}\right)\right)>
$$

where $\mathbf{q}=\mathbf{Q}-\mathbf{G}$ and $\mathbf{G}$ is the Bragg spot. $\hat{\alpha}$ is the unit vector along $\mathbf{G}+\mathbf{q}$. The summation of pairs of atoms is equivalently re-written as one over inter-atomic distances $\mathbf{R}=\mathbf{R}_{\mathbf{0} i}-\mathbf{R}_{\mathbf{0} j}$, so that Eq. 1 becomes the Fourier transform of projections of real space displacement-displacement auto-correlation tensor $\overleftrightarrow{C}(\mathbf{R})$ along $\alpha$ :

$$
I_{\text {diff }}(\mathbf{G}, \mathbf{q})=<\sum_{\mathbf{R}}(\alpha \cdot \overleftrightarrow{C}(\mathbf{R}) \cdot \alpha)|\mathbf{Q}|^{2} \cos (\mathbf{Q} \cdot \mathbf{R})>
$$




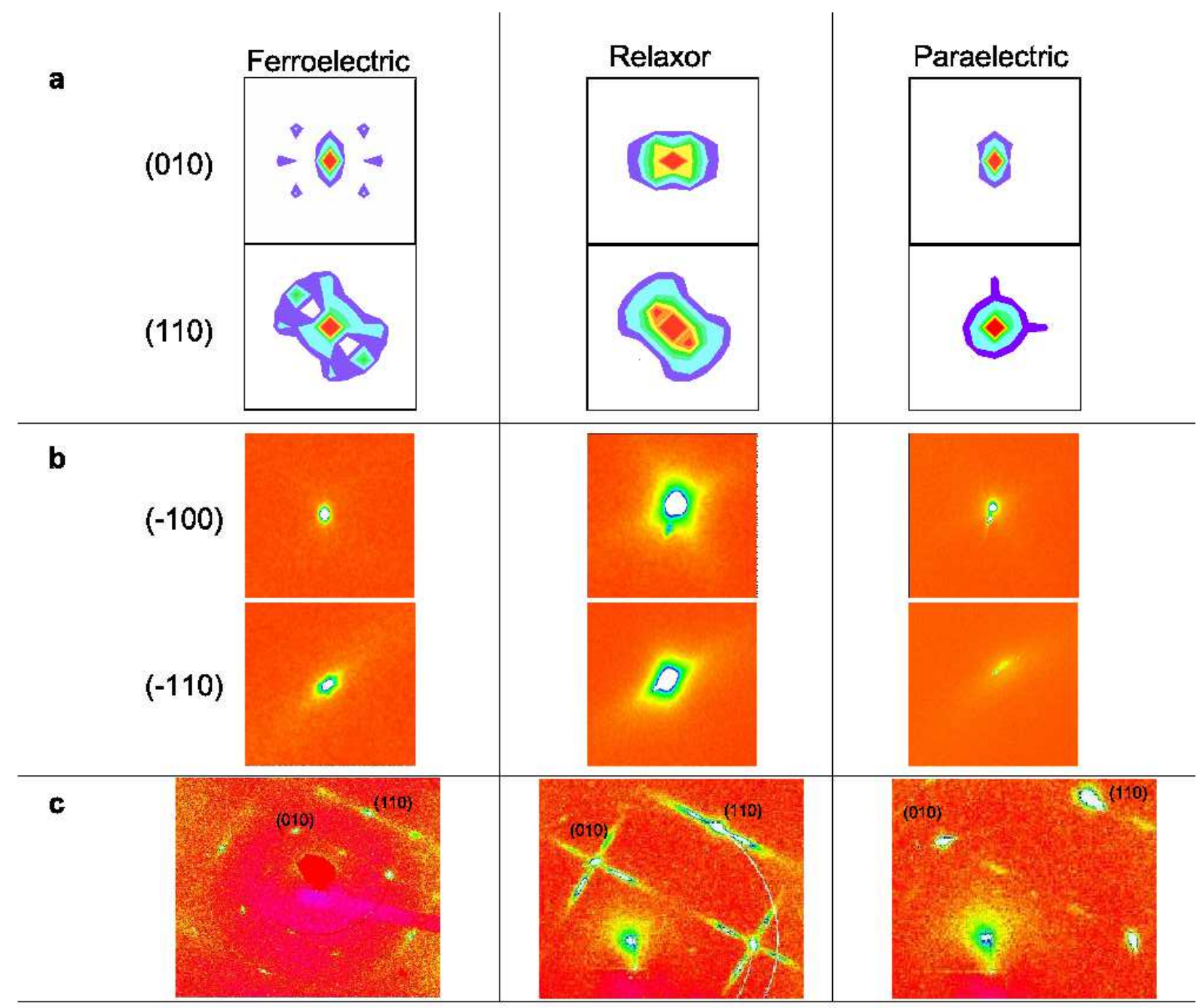

FIG. 2: Pressure dependence of diffuse scattering in simulations at $180 \mathrm{~K}$ (white background) and experiments (red background). (a) Simulated diffuse scattering in the ferroelectric $(\mathrm{P}=14 \mathrm{GPa})$, relaxor $(\mathrm{P}=18 \mathrm{GPa})$ and the paraelectric $(\mathrm{P}=25 \mathrm{GPa})$ phases respectively, show characteristic relaxor-phase butterfly and rod shaped diffuse scatting and radial diffuse scattering in the pressure induced paraelectric phase (b) Experiments at $300 \mathrm{~K}$ show weak diffuse scattering in the ferroelectric phase $(\mathrm{P}=0 \mathrm{GPa})$ but strong butterfly and rod shaped scattering in the relaxor phase $(\mathrm{P}=1.4 \mathrm{GPa})$. In the paraelectric phase $(\mathrm{P}=1.8 \mathrm{GPa})$ the butterfly and rod shapes disappear. (c) At $50 \mathrm{~K}$, we observe superlattice peaks in the ferroelectric phase $(\mathrm{P}=0.6 \mathrm{GPa})$. In the relaxor phase $(\mathrm{P}=2.7 \mathrm{GPa})$ on top of the butterfly and rod shapes we observe satellite spots. Further increase in pressure to $10 \mathrm{GPa}$ destroys the diffuse scattering, leading to a pressure induced relaxor to paraelectric phase transition. (The bright spot at the center of the image is due to the beam stopper.)

where $\overleftrightarrow{C}(\mathbf{R})$, given, in symmetric form is

$$
C_{\alpha \beta}(\mathbf{R})=\frac{1}{4} \sum_{i}\left(\xi_{i \alpha} \xi_{i \pm \mathbf{R} \beta}+\xi_{i \beta} \xi_{i \pm \mathbf{R} \alpha}\right),
$$

and the " \pm " notation implies summation over both signs.

For small $|\mathbf{q}|$, we approximate $\mathbf{G}+\mathbf{q}$ by $\mathbf{G}$ and $\hat{\alpha}$ by the unit vector $\hat{\alpha}_{\mathbf{G}}$. Fig 3 . shows the real space correlations in the relaxor phase $(180 \mathrm{~K}, 18 \mathrm{GPa})$ with projections along $\hat{\alpha}_{\mathbf{G}}=[100]$ and [110] summed over the ' $z$ ' direction as well as their Fourier-transforms. The diffuse scattering butterfly and rod shapes come from $\alpha_{\mathbf{G}}=[100]$ and $\alpha_{\mathbf{G}}=[110]$ projections, respectively, of the COR-COR real-space correlations. The CDR-CDR regions have strong correlation along the direction perpendicular to the radial direction, leading to the radial diffuse scattering in the Fourier space. In 3D (not shown) the [110] (as well as [111]) real space projections appear as ellipsoids, while the [100] correlations appear as discs. 


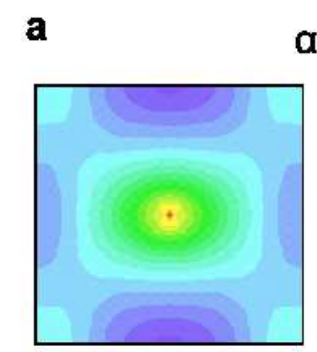

\section{b}

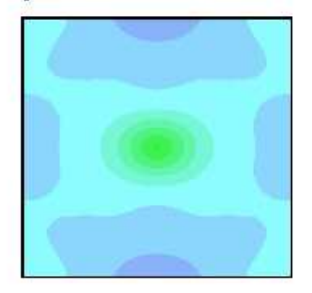

c

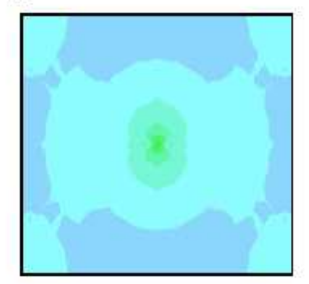

Real-space projection $a=[100]$
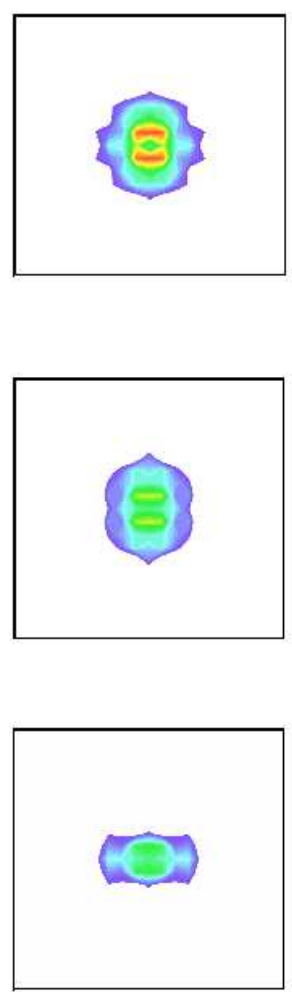

Fourier transform d $\quad \alpha=[110]$
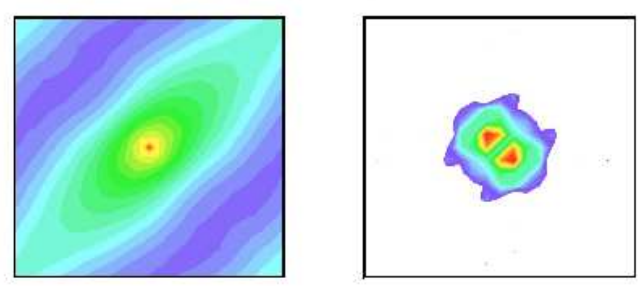

e
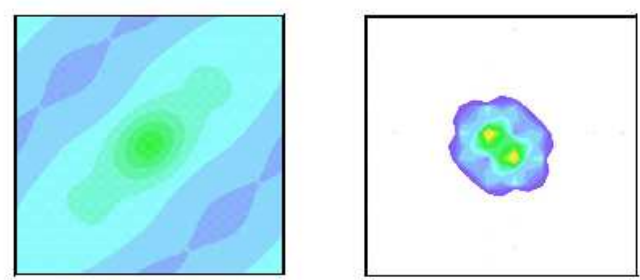

f

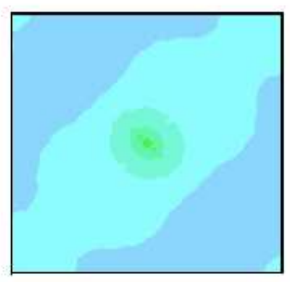

Real-space projection

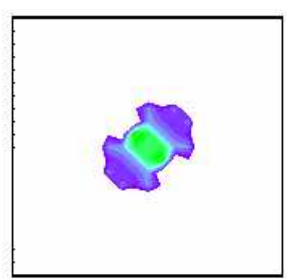

Fourier transform

FIG. 3: Real-space correlations (Eq. 3) projected along [100] and [110] directions and their direct Fourier transforms. (a) and (d) show correlations between all 'Pb' atoms, which reproduce the experimentally observed butterfly- and rod- shaped diffuse scattering. (b) and (e) show the correlations between COR-COR regions which are extended in the real space along directions normal to the butterfly and rod directions. Their Fourier transform gives the characteristic diffuse scattering. (c) and (f) show the correlations between the CDR-CDR regions which when Fourier transformed give the radial patterns

We conclude that PSN shows ferroelectric $\rightarrow$ relaxor $\rightarrow$ paraelectric phase transition with increase in temperature and/or pressure in our experiments. The relaxor phase in the experiments is characterized by butterfly and rod shaped diffuse scattering.

Simulations further reveal that the anisotropic correlations from COR-COR 'Pb' atoms which only have orientational degrees of freedom, give rise to the characteristic relaxor-phase diffuse scattering, rather than the effect of strain or artificial atomic shifts or domain boundaries. Polarization would be accompanied by local strain inhomogeneities, that would cause additional contribution to the diffuse scattering [11], but are a secondary effect. The radial diffuse scattering is identified as coming from local concentration fluctuations at the B-site, which reflect in the $\mathrm{Pb}$ displacements from corresponding fluctuations in local electric-fields on $\mathrm{Pb}$. Coulomb energy is minimized when the local polarization aligns parallel to the local field. Our result suggests that by engineering the shapes and relative positions of the COR regions, one could possibly control the anisotropy in the COR-COR correlations, leading to the design of new relaxor materials with superior electromechanical properties.

An increase in pressure reduces the ferroelectric (free energy) well depth and therefore the ferroelectric $\mathrm{Pb}$ displacements in COR. This reduces PNR-PNR correlations (and not their sizes) and hence the butterfly and rod shaped diffuse scattering. In the ferroelectric phase, the whole system, including the disordered matrix, has a uniform 
polarization along $<111>$ directions. This enhancement of the local polarization reduces the effect of correlated $\mathrm{Pb}$ displacements in COR-COR pairs, greatly diminishing the characteristic butterfly- and rod- shaped diffuse scattering features. With increase in temperature, thermal fluctuations of the polarization decrease the magnitude of the COR polarization, thereby decreasing the COR-COR correlations. Above $T_{d}$, only the CDR-CDR radial contributions remain.

The authors thank Stephen Gramsch and Eugene Venturini for their useful discussions. This work was sponsored by the Office of Naval Research under Grants No. N00014-07-1-0451 and N00014-02-1-0506; the Carnegie/Department of Energy Alliance Center (CDAC, DE-FC03-03NA00144). Use of the Advanced Photon Source was supported by the U. S. Department of Energy under Contract No. DE-AC02-06CH11357.

[1] S. E. Park and T. R. Shrout, Jpn. J. Appl. Phys. 82, 1804 (1997).

[2] G. A. Samara, Phys. Rev. Lett. 77, 314 (1996).

[3] G. A. Samara, Phys. Rev. B 71, 224108 (2005).

[4] G. Burns and F. H. Dacol, Solid State Commun. 48, 853 (1983).

[5] I.-K. Jeong, T. W. Darling, J. K. Lee, T. Proffen, R. H. Heffner, J. S. Park, K. S. Hong, W. Dmowski, and T. Egami, Phys. Rev. Lett. 94, 147602 (2005).

[6] G. Xu, Z. Zhong, H. Hiraka, and G. Shirane, Phys. Rev. B 70, 174109 (2004).

[7] M. Ahart, R. E. Cohen, V. Struzhkin, E. Gregoryanz, D. Rytz, S. A. Prosandeev, H.-k. Mao, and R. J. Hemley, Phys. Rev. B 71, 144102 (2005).

[8] P.-E. Janolin, B. Dkhil, P. Bouvier, J. Kreisel, and P. A. Thomas, Phys. Rev. B 73, 094128 (2006).

[9] P. Gehring, H. Hiraka, C. Stock, S.-H.Lee, W. Chen, Z.-G. Ye, S. B. Vakhrushev, and Z. Chowdhuri, Phy. Rev. B 79, 224109 (2009).

[10] M. Paściak, M. Wolcyrz, and A. Pietraszko, Phys. Rev. B 76, 014117 (2007).

[11] S. Vakhrushev, A. Ivanov, and J. Kulda, Phys. Chem. Chem. Phys. 7, 2340 (2005).

[12] T. R. Welberry and D. J. Goossens, Acta Cryst. A 64, 23 (2008).

[13] S. Tinte, B. P. Burton, E. Cockayne, and U. V. Waghmare, Phys. Rev. Lett. 97, 137601 (2006).

[14] Y. Bing and Z. G. Ye, Crystal Growth 250, 118 (2003).

[15] U. Rutt, M. A. Beno, J. Strempfer, G. Jennings, C. Krutz, and P. A. Montano, Nucl. Instrum. Methods Phys. Res. A 467-468, 1026 (2001).

[16] Y. Feng, M. S. Somayazulu, R. Jaramillo, and T. F. Rosenbaum, Rev. Sci. Instrum. 76, 063913 (2005).

[17] S. Ravy, J.-P. Itie, A. Polian, and M. Hanfland, Phys. Rev. Lett. 99, 117601 (2007).

[18] C. A. Randall, S. A. Markgraf, A. S. Bhalla, and K. Baba-Kishi, Phys. Rev. B 40, 413 (1989).

[19] U. V. Waghmare and K. M. Rabe, Phys. Rev. B 55, 6161 (1997).

[20] W. Zhong, D. Vanderbilt, and K. M. Rabe, Phys. Rev. B 52, 6301 (1995).

[21] B. P. Burton, E. Cockayne, and U. V. Waghmare, Phys. Rev. B 72, 064113 (2005).

[22] Simulations at each pressure-temperature were performed on a $40 \times 40 \times 40$ supercell that contained 32000 atoms for about 300 ps with a time step of 0.6 fs using a Nose thermostat. The barostat only coupled to the homogeneous strain. Intensitites were computed after an equilibration time of $150 \mathrm{ps}$.

[23] H. Krakauer, R. Yu, C.-Z. Wang, K. M. Rabe, and U. V. Waghmare, J. Phys.: Condens. Mater 11, 3779 (1999). 